\title{
Hypoxia and Polyionotrope Induced Junctional Ectopic Tachycardia
}

\author{
Debasis Acharya, Debasish Das, Jaideep Das Gupta, Subhas Pramanik \\ Department of Cardiology, All India Institute of Medical Sciences (AIIMS), Bhubaneswar, 751019, India.
}

\begin{abstract}
Corresponding Author:
Dr Debasis Acharya

Email: debasisacharyabhu@gmail.com

This is an Open Access article distributed under the terms of the Creative Commons Attribution License (creativecommons.org/ licenses/by/3.0).
\end{abstract}

Received : February 19, 2021

Accepted : July 12, 2021

Published : August 20, 2021

\begin{abstract}
Background: Junctional ectopic tachycardia (JET) is one of the rare supraventricular tachycardia seen in infancy and childhood. Case Report: We report a rare case of hypoxia and polyionotrope induced JET in a 11-year-old child with Ewing sarcoma admitted with septic shock. Conclusion: Ionotropes can accelerate any myofibre but this is a rare case where polyionotropes accelerated the atrioventricular junction (AV junction) resulting in incessant JET.
\end{abstract}

Keywords: Arrhythmia, Ectopic Junctional Tachycardia, Ewing Sarcoma, Septic Shock, Supraventricular Tachycardia.

\section{Introduction}

Tachyarrythmia originating from atrioventricular (AV) node, AV junction or proximal bundle of His is known as junctional tachycardia (JT) or junctional ectopic tachycardia (JET) which commonly occurs in infancy and childhood [1]. Broadly JT can be congenital or acquired. Postoperative JET is the most common acquired cause: hypoxia, dyselectronemia, digoxin toxicity are rare causes of junctional ectopic tachycardia. We report a rare case of JET where polyionotropes increased $\mathrm{AV}$ junction automaticity resulting in incessant JET.

\section{Case Report}

A 11-year-old male child with Ewing sarcoma and structurally normal heart was admitted with septic shock and desaturation to the pediatric intensive care unit. He was mechanically ventilated and started with ionotropes (dopamine and noradrenaline and adrenaline) along with empirical injectable antibiotics after obtaining blood culture. Next day, patient developed narrow complex regular tachycardia suggestive of junctional ectopic tachycardia (JET). Child developed acute kidney injury with serum creatinine of $1.6 \mathrm{mg} /$ dL with normal serum $\mathrm{Na}^{+}$and $\mathrm{K}^{+}$level. Rapid bolus adenosine injection did not terminate the tachycardia. Patient was started with amiodarone infusion which reverted the rhythm after 6 hours of infusion. JET are sometimes incessant and also demonstrate even delayed response to broad spectrum anti-arrhythmic like amiodarone.

\section{Discussion}

Junctional ectopic tachycardia is a focal automatic tachycardia arising from $\mathrm{AV}$ node, $\mathrm{AV}$ junction or proximal His bundle. It occurs most commonly in infancy and childhood. Congenital form is rare, difficult to treat and associated with significant mortality [2]. Most common acquired JET is postoperative and occurs in 1 to $15 \%$ of pediatric

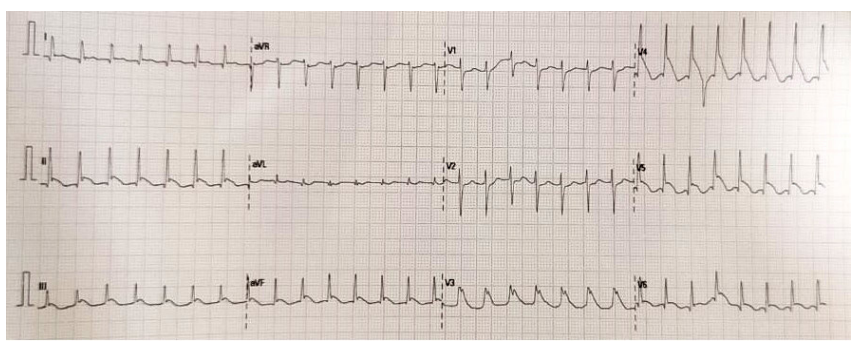

Fig.1: ECG showing junctional ectopic tachycardia (JET). 
cardiac surgery patients within 72 hours. Age less than 6 months, post-operative use of dopamine or milrinone, prolonged use of aortic cross-clamp and cardiopulmonary bypass, total surgical time, fever and dyselectronemia are the risk factors behind generation of JET [3-5]. It occurs more commonly in cardiac surgery that involves the crux of the heart including tetralogy of Fallot, AV canal, and ventricular septal defect repair, as well as repair of anomalous pulmonary venous return, arterial switch operation, Norwood procedure, and interrupted aortic arch repair. Fluid and electrolyte shifts, trauma, stretch, local edema, or ischemia in the region of the AV node or bundle of His are the proposed mechanism for post-operative JET.

Beside all those proposed mechanisms, we report a rare case of incessant JET that developed after polyionotrope infusion suggesting the fact that ionotropes can also rarely increase automaticity of $\mathrm{AV}$ junction resulting in this incessant JET.

\section{Conclusion}

We report a rare case of polyionotrope induced junctional ectopic tachycardia in an adolescent child reminding us the fact that judicious use of multiple ionotropes in children may save a child from developing this incessant rhythm. Usually associated with hemodynamic deterioration, early reversal of this rhythm improves the hemodynamics and clinical outcome.

Contributors: DA, DS: manuscript writing, patient management; JDG, SP: manuscript editing, patient management. DA will act as a study guarantor. All authors approved the final version of this manuscript and are responsible for all aspects of this study.

Funding: None; Competing interests: None stated.

\section{References}

1. Mildh L, Hiippala A, Rautiainen P, Pettilä V, Sairanen H, Happonen JM. Junctional ectopic tachycardia after surgery for congenital heart disease: incidence, risk factors and outcome. Eur J Cardiothorac Surg. 2011;39(1):75-80.

2. Coumel P, Fidelle JE, Attuel P, Brechenmacher C, Batisse A, Bretagne J, et al. Congenital bundle-of-his focal tachycardia. Cooperative study of 7 cases. Arch Mal Coeur Vaiss. 1976;69(9):899-909.

3. Paluszek C, Brenner P, Pichlmaier M, Haas NA, DallaPozza R, Hagl C, et al. Risk factors and outcome of post Fallot repair junctional ectopic tachycardia (JET). World J Pediatr Congenital Heart Surg. 2019;10(1):50-57.

4. Zampi JD, Hirsch JC, Gurney JG, Donohue JE, Yu S, LaPage MJ, et al. Junctional ectopic tachycardia after infant heart surgery: incidence and outcomes. Pediatr Cardiol. 2012;33(8):1362-1369.

5. Kean AC, Hazle M, LaPage MJ, Bromberg BI. Junctional tachycardia: congenital, acquired, postoperative In: Macdonald D, II, editor. Clinical cardiac electrophysiology in the young. $2^{\text {nd }}$ ed New York: Springer, 2016; pp. 157-169. 